\title{
49 HIGHLY MULTIPLEXED DETECTION OF CRITICAL IMMUNE CHECKPOINTS AND IMMUNE CELL SUBTYPES IN CANCEROUS FFPE TISSUES USING CODEX
}

Olive Shang*, Judi Gordon, Nadya Nikulina*, Sejal Mistry, Jasmine Singh, Hailing Zong, Jessica Yuan, Trillium Blackmer, Darren Locke, Oliver Braubach, Julia Kennedy-Darling, Peter Miller. Akoya Biosciences, Menlo Park, CA, USA

Background There is growing consensus that spatial biology is the key to unlocking the underlying mechanisms of cancer immunotherapy and to predicting patient outcomes. Indeed, a recent example using the Akoya Phenoptics technology revealed a unique phenotypic signature of CD8/Foxp3 positive cells embedded within the tumor microenvironment of patients that responded favorably to PD-1 checkpoint inhibition. ${ }^{1}$ In this case, the combination of both multiparameter and spatial readouts was required to correlate significantly with outcome. As the number of treatment options expands and knowledge regarding cell types that contribute to treatment mechanisms improves, so too do the number of markers required to analyze responses that enable discovery of new signatures.

Methods Here, we present data from the analysis of human FFPE cancer tissues using an expanded CODEX antibody catalog targeting a variety of immune, immune checkpoint and transcription factors. CODEX enables the highly multiplexed detection of more than 40 targets within the same tissue sample, with single cell resolution and without degradation of the sample.

Results Our expanded target list enables detection of key macrophage populations, $\mathrm{T}$ and $\mathrm{B}$ cell subtypes, granulocytes, dendritic cells, natural killer cells, stromal, tumor and epithelial cells. Additionally, the activation state of these immune and tumor cell types can be measured through detection of key immune checkpoints, including PD-1 and PD-L1. Through the addition of these critical markers, both cells known to contribute to treatment outcome and new biomarker signatures can be identified.

Conclusions Continued expansion of spatial biology discovery capabilities will be critical to continuing to improve patient outcomes and to develop new treatment options of solid tumors using cancer immunotherapies.

\section{REFERENCE}

1. Berry $S$, Taube J, et al. Analysis of multispectral imaging with the AstroPath platform informs efficacy of PD-1 blockade. Science. 2021; 372: 6547.

http://dx.doi.org/10.1136/jitc-2021-SITC2021.049 